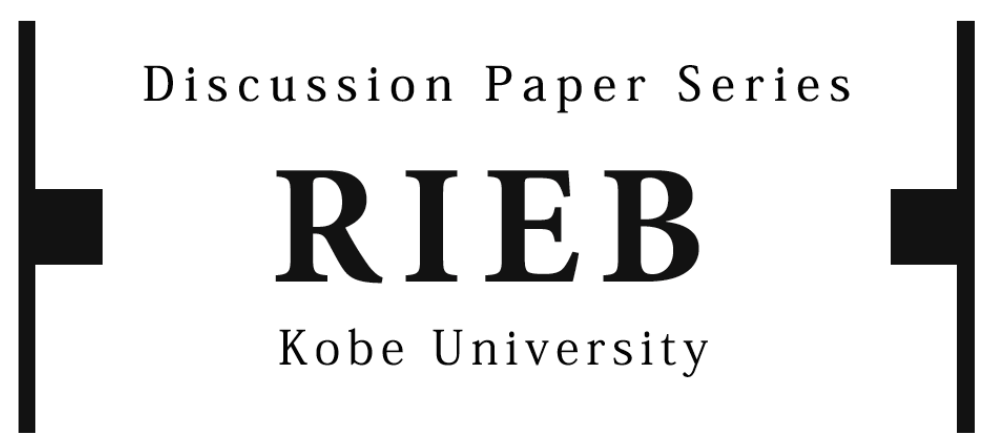

DP2015-28

Robust Predictions under Finite Depth of Reasoning

RIEB Junior Research Fellow

Kota MURAYAMA

July 3, 2015

* This Discussion Paper won the Kanematsu Fellowship Prize (FY 2013).

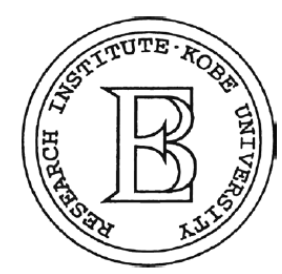

Research Institute for Economics and Business Administration Kobe University

2-1 Rokkodai, Nada, Kobe 657-8501 JAPAN 


\title{
Robust predictions under finite depth of reasoning
}

\author{
Kota Murayama*
}

June, 2015

\begin{abstract}
When players have a finite depth of reasoning, it is usually assumed that each player has a commonly known anchor behavior. This paper provides a general framework to examine whether predictions are robust to uncertainty about other players' anchors. We give two different sufficient conditions for the robustness. The first condition shows that any $\mathbf{p}$-dominant equilibrium is robust if players put sufficiently small probability (decreasing in $\mathbf{p}$ ) on highdepth types. This result highlights a distinction between two prominent finite depth of reasoning models: a risk dominated equilibrium is robust in the cognitive hierarchy model, but not in the level-k model. We also show that equilibria of dominance solvable models are robust.
\end{abstract}

Keywords: Robustness, iterative reasoning, level-k model, cognitive-hierarchy model, higher-order belief, bounded rationality.

${ }^{*}$ Department of Economics, Northwestern University. E-mail: murayama0209@gmail.com. I am most grateful to Takashi Ui for his guidance and feedback that have improved this paper in numerous aspects. Special thanks are to Atsushi Kajii and Daisuke Oyama for their time, advice, and encouragement. For helpful comments, I also thank Vincent Crawford, Hideshi Itoh, Michihiro Kandori, Willemien Kets, Takashi Kunimoto, Takeshi Nishimura, Ariel Rubinstein, and Satoru Takahashi, and seminar and conference participants at various places. This research is partially supported by Research Fellowships of Japan Society for the Promotion of Science for Young Scientists, and is awarded the 2014 Kanematsu Fellowship from Research Institute for Economics and Business Administration (RIEB) at Kobe University. 


\section{Introduction}

Consider the 11-20 money request game in Arad and Rubinstein (2012). In this game, each of two players requests an amount of money between 11 and 20 shekels. Each player receives the requested amount and if he requests exactly one number less than the other player, he will receive an additional 20 shekels. Players typically solve this game by using inductive reasoning. If a player's initial guess of the opponent's request is 20 , then his best response is 19 . But if the opponent knows his initial guess and knows that he plays a best response to the guess, then she will choose 18 . In turn, if he knows this, he will choose 17 instead of 19.

One of the most prevalent models that features inductive reasoning is a finite depth of reasoning (FDR) model such as the level-k and cognitive-hierarchy models (Stahl and Wilson, 1994; Nagel, 1995; Camerer et al., 2004). This model makes two distinctive assumptions. First, each player has an anchor behavior and it is commonly known. ${ }^{1}$ Second, each player has a reasoning level as his type, and believes that other players have lower reasoning levels. ${ }^{2}$ Then we can define an inductive solution for each game: level-1 (L1) types best respond to the anchor, level-2 (L2) types best respond to a combination of the anchor and the best responses of L1 types, and so forth.

In the FDR model, each player's anchor behavior plays an important role as a starting point for other players' iterative reasoning. Unfortunately, it is rare to have a unique candidate for the anchor behavior even in simple games. For instance, while a uniformly random choice is commonly regarded as an anchor in the $2 / 3$ guessing game, a recent paper by Burchardi and Penczynski (2014) shows that players' beliefs about the anchor are concentrated around salient numbers such as 50, 66, and 100. Thus, it is essential for expanding the domain of applicability of FDR models to

\footnotetext{
${ }^{1}$ Anchor behavior is called level-0 action in the experimental literature. Our use of terminology intends to emphasize that the anchor behavior is a characteristic of underlying games, and is a different object from player's FDR.

${ }^{2} \mathrm{~A}$ difference between level-k and cognitive-hierarchy models is their specification of beliefs about other players' reasoning levels. Each type in the level-k model believes that other players have one lower reasoning level, and in the cognitive-hierarchy model, each type's belief is a conditional probability distribution induced by a common probability distribution over reasoning levels.
} 
know how player's behavior differs if the anchor behavior is not common knowledge.

This paper provides a general framework to analyze the robustness of predictions to small uncertainty about an anchor behavior. From the perspective of an analyst, we have the following scenario in mind. An analyst is almost certain that a particular FDR model describes the relevant strategic environment; however, she suspects that players might consider other possibilities of anchor behaviors. Under this situation, if the prediction is known to be robust, any model with uncertainty has the approximately same prediction as long as possible anchor behaviors are close to the original one with high probability.

Our main contribution is giving a theoretical foundation for using FDR models in general environments. ${ }^{3}$ We provide an upper-bound on beliefs about other players' reasoning levels that makes a given prediction robust. For instance, consider a FDR model whose underlying game is given by a two-player, two-action game with two strict Nash equilibria. We show that the risk dominated prediction, in which every type follows the risk dominated Nash equilibrium, is robust in the cognitive-hierarchy model but not in the level-k model. This result highlights a distinction between the two prominent FDR models in the sensitivity of predictions to the violation of the common knowledge assumption.

More formally, a (FDR) model is a combination of a game with anchor behavior (a game for short) in which each player is given an anchor behavior, and a set of reasoning levels (or types). An equilibrium of this model is described above. Our robustness test proceeds as follows: Fix a model and its equilibrium $s^{*}$. We embed this model by introducing incomplete information about its anchor behavior. The embedding model is said to be close to the original model if the anchor behavior remains the same and each player knows that with high probability. ${ }^{4}$ We say that $s^{*}$ is robust if each close embedding model has an equilibrium in which $s^{*}$ is played with high probability.

\footnotetext{
${ }^{3}$ There has been a growing number of papers that introduce FDR to various kinds of games including auctions (Crawford and Iriberri, 2007; Crawford et al., 2009), global games (Kneeland, 2014), and mechanism design (Crawford, 2015; de Clippel et al., 2015).

${ }^{4}$ In the embedding model, players have a common prior on anchor behaviors. The closeness is measured by this prior.
} 
The first sufficient condition utilizes a relation between the robustness and beliefs about other players' reasoning levels. We define a $\boldsymbol{p}=\left(p_{1}, \ldots, p_{I}\right)$-dominant equilibrium analogously to the standard $\mathbf{p}$-dominance. ${ }^{5}$ We show that any $\mathbf{p}$-dominant equilibrium is robust if there exists a threshold level $\bar{c}$ such that any higher-level type of players than $\bar{c}$ puts sufficiently small probability (decreasing in $\mathbf{p}$ ) on his opponents having levels no lower than $\bar{c}$. As a corollary, a p-dominant equilibrium in the level-k model is shown to be robust if $p_{i}<1 / I$ for each player $i$, and any $\mathbf{p}$-dominant equilibrium with $\mathbf{p} \in[0,1)^{I}$ is robust in the cognitive-hierarchy model for any common distribution.

For the second result, we say a model is dominance solvable if the model yields a unique action for sufficiently high-level types through the iterated deletion of never best responses. We show that a strict equilibrium of the dominance solvable model is robust. Particularly, the level-k model is dominance solvable if and only if its underlying game is dominance solvable in the usual sense. Hence, the level-k model with a dominance solvable game gives a robust prediction generically in payoffs.

Our notion of robustness is based on Kajii and Morris (1997), who study the robustness of equilibria to a violation of common knowledge about payoffs (hereafter, KM robustness). The main difference is that while they consider a complete information game and introduce payoff uncertainty, we start from the FDR model and introduce uncertainty in anchor behavior. As a result, since the anchor works as a coordination device for high-level types under a certain condition on beliefs, a weaker sufficient condition can be obtained than that for KM robustness.

Strzalecki (2014) also derives a similar bound on beliefs in Rubinstein's (1989) email game. He shows that coordination is achieved after exchanging a finite number of messages if the player's belief on high-level types is bounded. Thus, a particular specification of beliefs makes the strategic behavior of high-level types change in a uniformly continuous way in the specific game. Although our question and method are different, our result further develops this insight. Especially, while Strzalecki's bound depends on details of the email game, our bound uniformly holds for general

\footnotetext{
${ }^{5} \mathrm{~A}$ strategy profile is $\mathbf{p}$-dominant if it is optimal for each type of each player $i$ to play the specified strategy whenever he believes consistently with his belief about others' types that the other players will follow the specified strategies with probability at least $p_{i}$.
} 
games, and is expressed as a function of the "strength" of equilibrium and the number of players.

The rest of the paper is organized as follows: In Section 2, we discuss the relations of this paper to other studies. Section 3 introduces our framework. Section 4 presents a motivating example. In Sections 5 and 6 , we provide the two sufficient conditions. Section 7 discusses some limitation and future direction of our research. All proofs are shown in the Appendix.

\section{Related Literature}

This paper is related to two strands of literature. A role of anchor behavior in a strategic environment is pioneered by Schelling (1960). In the experimental literature, a modeler's choice of an anchor has received increasing attention. ${ }^{6}$ Both Burchardi and Penczynski (2014) and Agranov et al. (2015) identify the anchor behavior of the $2 / 3$ guessing game, but lead to different conclusions. Heap et al. (2014) test a saliency-based anchor behavior by using games in which one action has a uniquely different label from the others. Based on the observation that players do not necessarily best respond to the salient anchor, they raise a doubt on its universality. $^{7}$ In a related context, Kneeland (2015) proposes a way of identifying player's depth of reasoning independently of the specification of anchor behaviors.

The present paper studies a behavioral implication of not assuming the common knowledge of anchor behaviors. For this purpose, we use techniques from the robustness literature, especially from Kajii and Morris (1997). There has been many follow-up papers that propose a variety of sufficient conditions for KM robustness. Ui (2001) shows that a unique maximizer of the potential function is KM robust. This approach is further elaborated by Morris and Ui (2005), Oyama and Tercieux (2009), and Nora and Uno (2014). Other sufficient conditions include a unique correlated equilibrium (Kajii and Morris, 1997) and p-best response sets (Tercieux, 2006). Investigating corresponding notions of these conditions in the FDR model might be interesting, but it is beyond the scope of this paper.

\footnotetext{
${ }^{6}$ Crawford et al. (2013) and Camerer and Ho (2014) provide an excellent survey of FDR models.

${ }^{7}$ See also comments by Crawford (2014).
} 
Finally, Heifetz and Kets (2013) also consider the robustness of predictions when players can have either infinite or finite depth of reasoning. They construct a general universal type space that contains both the standard universal type space (Mertens and Zamir, 1985; Brandenburger and Dekel, 1993) and a universal cognitive type space (Strzalecki, 2014). In contrast to Weinstein and Yildiz (2007), they show that in generic global games (Carlsson and van Damme, 1993), there are types that robustly have multiple rationalizable actions when players' infinite depth of reasoning is "almost" common knowledge. The main difference between their approach and ours is that while they use the weakest solution concept and establish its robustness focusing on global games, we pay more attention to a refined solution concept, and derive its robustness in general games.

\section{Framework}

\subsection{Game with anchor behavior}

A game with anchor behavior (called a game hereafter) is a tuple $\mathcal{G}=\left(\mathcal{I},\left\{A_{i}, u_{i}, f_{i}\right\}_{i \in \mathcal{I}}\right)$, where $\mathcal{I}=\{1,2, \ldots, I\}$ is a set of players, $A_{i}$ is a finite set of actions for player $i$, and $u_{i}: A \rightarrow \mathbb{R}$ is player $i$ 's payoff function, where $A=A_{1} \times \cdots \times A_{I}$. Unlike the standard complete information game, $f_{i} \in A_{i}$ specifies an anchor behavior for player $i{ }^{8}$ This anchor can be interpreted as a focal point or a naive play of the game, which is commonly known among players. It seems difficult in general to find an anchor if it exists, however, the following two games have a somewhat clear candidate for the anchor.

Example 3.1. (Salient label in a symmetric coordination game)

Each of two players chooses one of the two locations: the Sears Tower (a recognizable Chicago landmark) or the little-known AT\&T Building. Players will receive $\$ 100$ if both choose the same location, and will receive nothing if they choose different locations. Payoffs are shown in Table 1. In this game, the salience of the Sears

\footnotetext{
${ }^{8}$ While it is conceptually easy to incorporate mixed anchor behaviors, we focus on pure anchor behaviors to keep our notation as simple as possible.
} 


\begin{tabular}{lcc}
\hline \hline & Sears Tower & AT \& T \\
\hline Sears Tower & 100,100 & 0,0 \\
\hline AT \& T & 0,0 & 100,100 \\
\hline
\end{tabular}

Table 1: Payoff matrix of the symmetric coordination game.

Tower may work as an anchor. In fact, Crawford et al. (2008) report that in their experiments, about $90 \%$ of subjects chose the Sears Tower.

Example 3.2. (Random play in a $2 / 3$-guessing game)

Each of $I$ players calls an integer $a_{i} \in[0,100]$, and his payoff is given by

$$
u_{i}\left(a_{1}, \ldots, a_{I}\right)= \begin{cases}1 & \text { if } a_{i}=\left[2 / 3 \cdot \bar{a}_{-i}\right] \\ 0 & \text { if otherwise }\end{cases}
$$

where $\bar{a}_{-i}=\left(a_{1}+\cdots+a_{i-1}+a_{i+1}+\cdots+a_{I}\right) /(I-1)$, and $[a]$ is the closest integer to $a$. Thus, each player has a strict incentive to guess the average of other players' choices $\bar{a}_{-i}$, and call the closest integer to $2 / 3 \cdot \bar{a}_{-i}$. Agranov et al. (2015) find that naive players tend to choose 50 on average, and thus the random play seems a candidate for the anchor in this game.

\subsection{Cognitive type space}

To model player's FDR, we follow the formulation of Strzalecki (2014). A (cognitive) type space of player $i$ is given by $\mathcal{T}_{i}=\left(C_{i}, \mu_{i}\right)$, where $C_{i}=\{0,1,2, \ldots\}$ is a set of types, and $\mu_{i}: C_{i} \backslash\{0\} \rightarrow \Delta\left(C_{-i}\right)$ is player $i$ 's belief about other players' types, where $C_{-i}=C_{1} \times \cdots \times C_{i-1} \times C_{i+1} \times \cdots \times C_{I}$, and $\Delta\left(C_{-i}\right)$ denotes a collection of probability measures on $C_{-i}$. We write $\mu_{i}\left(c_{i}\right)\left(c_{-i}\right)$ for the probability of the singleton event $\left\{c_{-i}\right\}$ given $c_{i}$, where $c_{-i}=\left(c_{1}, \ldots, c_{i-1}, c_{i+1}, \ldots, c_{I}\right)$. We assume that each type believes that other players have lower types: $\mu_{i}\left(c_{i}\right)\left(\left\{c_{-i} \in C_{-i}: c_{j}<c_{i}\right.\right.$ for each $j \neq i\})=1$ for each $c_{i} \in C_{i} \backslash\{0\}$. Since each type is naturally identified with a reasoning level, a player of type $\mathrm{k}$ is called Lk type. Note that L0 types are "seeds" for reasoning, and used to model a starting point for inductive reasoning. Let us call $\mathcal{T}=\mathcal{T}_{1} \times \cdots \times \mathcal{T}_{I}$ a (cognitive) type space. The following two are the leading examples of $\mathcal{T}$. 
Example 3.3. (Level-k type space)

The level-k type space, denoted by $\mathcal{T}^{k}$, is a type space in which each type believes that all the other players have exactly one lower level. Formally, for each $i \in \mathcal{I}$ and $c_{i} \in C_{i} \backslash\{0\}$,

$$
\mu_{i}\left(c_{i}\right)\left(c_{-i}\right)= \begin{cases}1 & \text { if } c_{j}=c_{i}-1 \text { for each } j \neq i \\ 0 & \text { if otherwise }\end{cases}
$$

Example 3.4. (Cognitive hierarchy type space)

The cognitive hierarchy $(\mathrm{CH})$ type space, denoted by $\mathcal{T}^{c}$, is a type space in which each type's belief is a conditional probability induced by a common distribution. Formally, there exists $\lambda \in \Delta\left(\mathbb{Z}_{+}\right)$with $\lambda(0)>0$ such that for each $i \in \mathcal{I}$ and $c_{i} \in C_{i} \backslash\{0\}$,

$$
\mu_{i}\left(c_{i}\right)\left(c_{-i}\right)=\left\{\begin{array}{cl}
\frac{\times_{j \neq i} \lambda\left(c_{j}\right)}{\left(\sum_{l=0}^{c_{i}-1} \lambda(l)\right)^{I-1}} & \text { if } c_{j}<c_{i} \text { for each } j \neq i \\
0 & \text { if otherwise. }
\end{array}\right.
$$

For instance, Camerer et al. (2004) use a Poisson distribution for $\lambda$.

\subsection{Solution concept}

Let us call $(\mathcal{G}, \mathcal{T})$ a $(F D R)$ model. Player $i$ 's (pure) strategy in $(\mathcal{G}, \mathcal{T})$ is a mapping $s_{i}: C_{i} \rightarrow A_{i}$ with $s_{i}(0)=f_{i}$. Let $S_{i}$ denote the set of player $i$ 's strategies for each $i \in \mathcal{I}$, and write $S=S_{1} \times \cdots \times S_{I}$ and $S_{-i}=S_{1} \times \cdots \times S_{i-1} \times S_{i+1} \times \cdots \times S_{I}$. In equilibrium of $(\mathcal{G}, \mathcal{T})$, L1 types play a best response to the anchor, and L2 types play a best response to the mix of the anchor and L1 type's best response according to his belief. Equilibrium behavior of higher-level types is similarly defined.

Definition 3.1. A strategy profile $s^{*}=\left(s_{1}^{*}, \ldots, s_{I}^{*}\right)$ is an equilibrium of $(\mathcal{G}, \mathcal{T})$ if, for each $i \in \mathcal{I}, c_{i} \in C_{i} \backslash\{0\}$, and $a_{i} \in A_{i} \backslash\left\{s_{i}^{*}\left(c_{i}\right)\right\}$,

$$
\sum_{c_{-i} \in C_{-i}}\left(u_{i}\left(s_{i}^{*}\left(c_{i}\right), s_{-i}^{*}\left(c_{-i}\right)\right)-u_{i}\left(a_{i}, s_{-i}^{*}\left(c_{-i}\right)\right)\right) \mu_{i}\left(c_{i}\right)\left(c_{-i}\right) \geq 0,
$$

where $s_{-i}^{*}\left(c_{-i}\right)=\left(s_{1}^{*}\left(c_{1}\right), \ldots, s_{i-1}^{*}\left(c_{i-1}\right), s_{i+1}^{*}\left(c_{i+1}\right), \ldots, s_{I}^{*}\left(c_{I}\right)\right)$.

In particular, we say that a strategy profile is a strict equilibrium if it satisfies the above condition with strict inequalities. By definition, a strict equilibrium is 
always unique if it exists. For instance, in the $2 / 3$-guessing game with the level-k type space, if the anchor behavior is given by 50 , there exists a strict equilibrium in which L1 types choose 33, L2 types choose 22, and L3 types choose 15, and so on.

\subsection{Robustness}

Our robustness concept is based on Kajii and Morris (1997). We first extend a model to accommodate situations where each player knows other player's anchor behavior only with high probability. An embedding game of $\mathcal{G}$ is a tuple $\mathcal{U}=$ $\left(\mathcal{I}, \Theta, P,\left\{A_{i}, u_{i}, \Pi_{i}, g_{i}\right\}_{i \in \mathcal{I}}\right)$, where $\Theta$ is a nonempty countable set of states, $P$ is a common prior on $\Theta$, and for each player $i \in \mathcal{I}, \Pi_{i}$ is a set of information partitions of $\Theta$, and $g_{i}: \Theta \rightarrow A_{i}$ is a state dependent anchor behavior of player $i$. We assume that $g_{i}$ is $\Pi_{i}$-measurable, that is, $g_{i}(\theta)=g_{i}\left(\theta^{\prime}\right)$ for each $\theta, \theta^{\prime} \in \pi_{i}$ and $\pi_{i} \in \Pi_{i}$. Note that $\Theta$ is uncertainty about the anchor behavior, and hence there is no payoff uncertainty in $\mathcal{U}$. We write $P(\theta)$ for the probability of the singleton event $\{\theta\}$ and $\pi_{i}(\theta)$ for the element of $\Pi_{i}$ containing $\theta$. Suppose that $P\left(\pi_{i}(\theta)\right)>0$ for each $\theta \in \Theta$ and $i \in \mathcal{I}$. Let $E(\mathcal{G})$ denote a set of all embedding games of $\mathcal{G}$, and let us call $(\mathcal{U}, \mathcal{T})$ an embedding model of $(\mathcal{G}, \mathcal{T})$.

An equilibrium of $(\mathcal{U}, \mathcal{T})$ is defined as each non-L0 type of player maximizing the expected payoff under his beliefs about other players' types and states. For each $i \in \mathcal{I}$, let us denote player $i$ 's (pure) strategy in $(\mathcal{U}, \mathcal{T})$ by $\sigma_{i}: C_{i} \times \Theta \rightarrow A_{i}$ with $\sigma_{i}(0, \theta)=g_{i}(\theta)$ for each $\theta \in \Theta$, and assume that $\sigma_{i}\left(c_{i}, \cdot\right)$ is $\Pi_{i}$-measurable for each $c_{i} \in C_{i} \backslash\{0\}$. We write $\sigma=\left(\sigma_{1}, \ldots, \sigma_{I}\right)$, and $\sigma_{-i}=\left(\sigma_{1}, \ldots, \sigma_{i-1}, \sigma_{i+1}, \ldots, \sigma_{I}\right)$. Then, the expected payoff of a strategy profile $\sigma$ for the Lk type of player $i$ at $\theta$ is

$$
U_{i}(\sigma, k, \theta) \equiv \sum_{c_{-i} \in C_{-i}} \sum_{\theta^{\prime} \in \Theta} u_{i}\left(\sigma_{i}(k, \theta), \sigma_{-i}\left(c_{-i}, \theta^{\prime}\right)\right) \mu_{i}(k)\left(c_{-i}\right) P\left(\theta^{\prime} \mid \pi_{i}(\theta)\right),
$$

where $\sigma_{-i}\left(c_{-i}, \theta^{\prime}\right)=\left(\sigma_{1}\left(c_{1}, \theta^{\prime}\right), \ldots, \sigma_{i-1}\left(c_{i-1}, \theta^{\prime}\right), \sigma_{i+1}\left(c_{i+1}, \theta^{\prime}\right), \ldots, \sigma_{I}\left(c_{I}, \theta^{\prime}\right)\right)$.

Definition 3.2. A strategy profile $\sigma^{*}$ is an equilibrium of $(\mathcal{U}, \mathcal{T})$ if, for each $i \in \mathcal{I}$, $c_{i} \in C_{i} \backslash\{0\}, \theta \in \Theta$, and $a_{i} \in A_{i}$,

$$
U_{i}\left(\sigma^{*}, c_{i}, \theta\right)-U_{i}\left(a_{i}, \sigma_{-i}^{*}, c_{i}, \theta\right) \geq 0 \text {. }
$$

Let us define an event $\Omega_{\mathcal{U}}$ in $\mathcal{U}$ in which each player's anchor behavior is specified as in $\mathcal{G}$, and each player knows that. That is, $\Omega_{\mathcal{U}} \equiv\left\{\theta \in \Theta: g_{-i}\left(\theta^{\prime}\right)=f_{-i}\right.$ for each 
$\theta^{\prime} \in \pi_{i}(\theta)$ and $\left.i \in \mathcal{I}\right\}$, where $g_{-i}\left(\theta^{\prime}\right)=\left(g_{1}\left(\theta^{\prime}\right), \ldots, g_{i-1}\left(\theta^{\prime}\right), g_{i+1}\left(\theta^{\prime}\right), \ldots, g_{I}\left(\theta^{\prime}\right)\right)$. We are now able to define a close embedding game to $\mathcal{G}$ in terms of the anchor behavior.

Definition 3.3. $\mathcal{U}$ is an $\varepsilon$-elaboration of $\mathcal{G}$ if $\mathcal{U} \in E(\mathcal{G})$ and $P\left(\Omega_{\mathcal{U}}\right)=1-\varepsilon$. Let $E(\mathcal{G}, \varepsilon)$ be the set of all $\varepsilon$-elaborations of $\mathcal{G}$.

In a 0 -elaboration, we have in particular that $\sigma_{i}(0, \theta)=f_{i}$ for each $\theta \in \Theta$ and $i \in \mathcal{I}$. Hence, for each equilibrium $s^{*}$ of $(\mathcal{G}, \mathcal{T})$, each $(\mathcal{U}, \mathcal{T})$ with $\mathcal{U} \in E(\mathcal{G}, 0)$ has an equilibrium $\sigma^{*}$ with $\sigma_{i}^{*}\left(c_{i}, \theta\right)=s_{i}^{*}\left(c_{i}\right)$ for each $\theta \in \Theta$ and $c_{i} \in C_{i}$.

In words, we say that an equilibrium of a model is robust (to incomplete information in anchor behavior) if any sufficiently "close" embedding model has an equilibrium whose action distribution is probabilistically close to the original equilibrium. For each strategy profile $\sigma$ of $(\mathcal{U}, \mathcal{T})$, let $P_{\sigma}(a \mid c)$ denote the probability of an action profile $a=\left(a_{1}, \ldots, a_{I}\right)$ being taken by a type profile $c=\left(c_{1}, \ldots, c_{I}\right)$, i.e., $P_{\sigma}(a \mid c)=P\left(\left\{\theta \in \Theta: \sigma_{i}\left(c_{i}, \theta\right)=a_{i}\right.\right.$ for each $\left.\left.i \in \mathcal{I}\right\}\right)$.

Definition 3.4. An equilibrium $s^{*}$ of $(\mathcal{G}, \mathcal{T})$ is robust if for each $\delta>0$, there exists $\bar{\varepsilon}>0$ such that any $(\mathcal{U}, \mathcal{T})$ with $\mathcal{U} \in E(\mathcal{G}, \varepsilon)$ has an equilibrium $\sigma^{*}$ that satisfies $P_{\sigma^{*}}\left(s^{*}(c) \mid c\right) \geq 1-\delta$ for each $c$ and $\varepsilon \leq \bar{\varepsilon}$, where $s^{*}(c)=\left(s_{1}^{*}\left(c_{1}\right), \ldots, s_{I}^{*}\left(c_{I}\right)\right)$.

There are two remarks on the definition of robustness. First, while we do not allow payoff uncertainty for simplicity, the same results follow as long as each $\mathcal{U} \in$ $E(\mathcal{G}, \varepsilon)$ has the same payoff structure with $\mathcal{G}$ and players know that in $\Omega_{\mathcal{U}}$. Second, each type's belief about others' types is assumed to be independent of his belief about states. Alternatively, we can endow each type with a belief $\rho_{i}\left(c_{i}\right) \in \Delta\left(C_{-i} \times \Theta\right)$ whose marginal distribution on $C_{-i}$ coincides with $\mu_{i}\left(c_{i}\right)$. Allowing such a correlation makes our robustness test more stringent.

\section{Motivating example}

We illustrate the intuition of our result by using a coordinated attack game. ${ }^{9}$ There are two players, and each player chooses either $\operatorname{Attack}(A)$ or $N$ ot $\operatorname{Attack}(N A)$. Payoffs are shown in Table 2. Suppose that $A$ is an anchor behavior for both players. In this game $\mathcal{G}, N A(A)$ becomes a unique best response for each player whenever

\footnotetext{
${ }^{9}$ This example is taken from Strzalecki (2014).
} 


\begin{tabular}{ccc}
\hline \hline & $A$ & $N A$ \\
\hline$A$ & 1,1 & $-2,0$ \\
\hline$N A$ & $0,-2$ & 0,0 \\
\hline
\end{tabular}

Table 2: Payoff matrix of the coordinated attack game.

he believes that his opponent will choose $N A(A)$ with probability more than $1 / 3$ $(2 / 3)$. Thus, $(N A, N A)$ is a risk dominant and $(A, A)$ is a risk dominated Nash equilibrium. Since $(A, A)$ is the anchor, $(\mathcal{G}, \mathcal{T})$ has a unique equilibrium in which every type plays $A$ regardless of the specification of $\mathcal{T}$. Let $s^{*}$ denote this risk dominated equilibrium.

\section{Case 1: Level-k type space}

First, consider the level-k type space $\mathcal{T}^{k}$. We show that $s^{*}$ is not robust in this model. Let us introduce an email game elaboration such that $\Theta=\{(0,0),(1,0),(1,1),(2,1), \ldots\}$, $\Pi_{1}=\{\{(0,0)\},\{(1,0),(1,1)\}, \ldots\}, \Pi_{2}=\{\{(0,0),(1,0)\},\{(1,1),(2,1)\}, \ldots\}$, and $P((a, b))=$ $\varepsilon(1-\varepsilon)^{a+b}$ for each $(a, b) \in \Theta .{ }^{10}$ Assume that each player's anchor behavior remains $A$ except for player 1's anchor behavior at $(0,0)$ which is now given by $N A$. Let $\mathcal{U}^{e}$ denote this $\varepsilon(2-\varepsilon)$-elaboration of $\mathcal{G}$. Table 3 shows each player's posterior belief about states.

\begin{tabular}{|c|c|c|c|c|c|}
\hline$\Pi_{1}$ & 1 & $\frac{1}{2-\varepsilon}$ & $\frac{1-\varepsilon}{2-\varepsilon}$ & $\frac{1}{2-\varepsilon}$ & $\frac{1-\varepsilon}{2-\varepsilon}$ \\
\hline$\Theta$ & $(0,0)$ & $(1,0)$ & $(1,1)$ & $(2,1)$ & $(2,2)$ \\
\hline$\Pi_{2}$ & $\frac{1}{2-\varepsilon}$ & $\frac{1-\varepsilon}{2-\varepsilon}$ & $\frac{1}{2-\varepsilon}$ & $\frac{1-\varepsilon}{2-\varepsilon}$ & $\ldots$ \\
\hline
\end{tabular}

Table 3: Posterior beliefs over $\Theta$.

In $\left(\mathcal{U}^{e}, \mathcal{T}^{k}\right), N A$ is a unique best response for the L1 type of player 2 in $\{(0,0),(1,0)\}$ since he believes with certain that player 1 will play $N A$ with probability $1 /(2-\varepsilon)$. In turn, $N A$ is played in $\{(1,0),(1,1)\}$ by the L2 type of player 1 since he believes that player 2 is of L1 type and will play $N A$ with probability $1 /(2-\varepsilon)$ (see Table 4). Repeating similar arguments, we can show that there exists

\footnotetext{
${ }^{10}$ This embedding game has an essentially same information structure with that of the email game.
} 
a type profile that plays $(N A, N A)$ with arbitrarily high probability in a unique equilibrium of $\left(\mathcal{U}^{e}, \mathcal{T}^{k}\right)$. Thus, we have shown that $s^{*}$ is not robust in $\left(\mathcal{G}, \mathcal{T}^{k}\right)$.

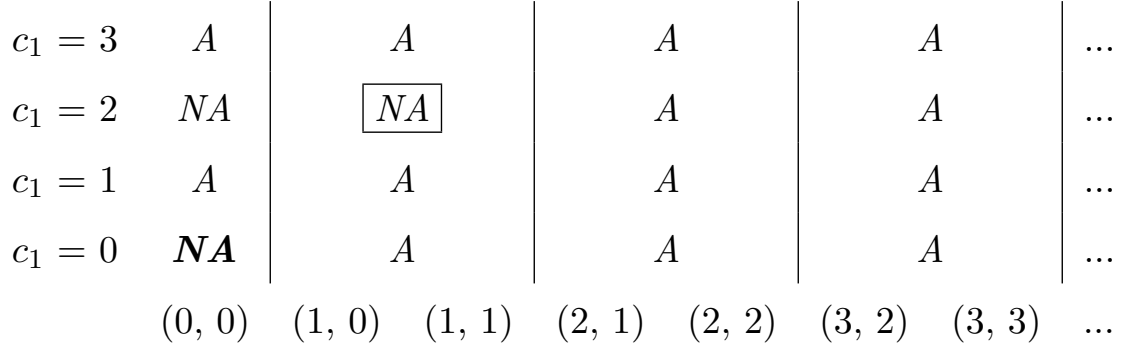

$$
\begin{aligned}
& \begin{array}{cc|c|c|c}
c_{2}=0 & A & A & A & \ldots \\
c_{2}=1 & N A & A & A & \ldots \\
c_{2}=2 & A & A & A & \ldots \\
c_{2}=3 & N A & N A & A & \ldots
\end{array}
\end{aligned}
$$

Table 4: Equilibrium actions of L1, L2, and L3 types in $\left(\mathcal{U}^{e}, \mathcal{T}^{k}\right)$.

\section{Case 2: CH type space}

Next, consider the $\mathrm{CH}$ type space $\mathcal{T}^{c}$ with a uniform distribution. We can show that $s^{*}$ is played with high probability in $\left(\mathcal{U}^{e}, \mathcal{T}^{c}\right)$. For instance, consider the equilibrium action of player 1's L2 type in $\{(1,0),(1,1)\}$. This type believes that player 2 is either L0 or L1 type equally likely and that player 2 will play $A$ with probability $1-1 / 2(2-\varepsilon)$. Hence, his unique best response in $\{(1,0),(1,1)\}$ is now given by $A$ for sufficiently small $\varepsilon$ (see Table 5 ). To formally show that $A$ is played by any type

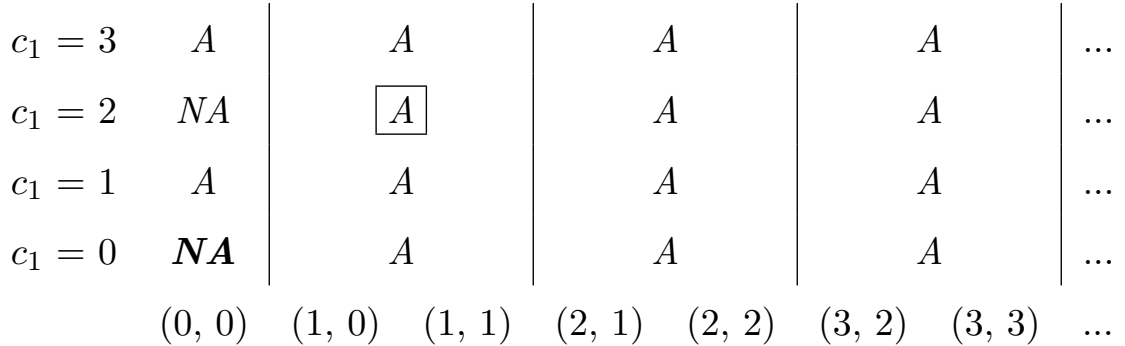

$$
\begin{aligned}
& \begin{array}{rlr}
c_{2} & =0 & A \\
c_{2}=1 & N A \\
c_{2}=2 & A \\
c_{2}=3 & N A
\end{array}
\end{aligned}
$$

\begin{tabular}{l|l}
$A$ & $A$ \\
$A$ & $A$ \\
$A$ & $A$ \\
$A$ & $A$
\end{tabular}

Table 5: Equilibrium actions of L1, L2, and L3 types in $\left(\mathcal{U}^{e}, \mathcal{T}^{c}\right)$.

with high probability, we use Theorem 4 of Strzalecki (2014): 
Theorem 4.1. (Theorem 4 of Strzalecki 2014)

Suppose $\mathcal{T}$ admits beliefs such that for each $i=1,2, \inf _{m} \mu_{i}\left(c^{m}\right)\left(\left\{c_{j}: c^{m-1} \leq c_{j}\right\}\right)<$ $(2-\varepsilon) / 3$ for any strictly increasing sequence $\left(c^{m}\right) \in \mathbb{N}^{\infty}$. Then there exists $n \in \mathbb{N}$ such that all types choose to Attack at any $\theta \geq(n, n)$.

Since $\mathcal{T}^{c}$ satisfies the above condition (for any common distribution), it follows that $A$ is played by all types with high probability as long as $\varepsilon$ is sufficiently small. Thus $s^{*}$ is played with high probability in $\left(\mathcal{U}^{e}, \mathcal{T}^{c}\right)$.

It is important to note that this result does not tell us whether $s^{*}$ is robust in $\left(\mathcal{G}, \mathcal{T}^{c}\right)$, since we must check all the other $\varepsilon$-elaborations to establish the robustness. Nevertheless, our main result states that a different specification of type spaces yields a different sufficient condition for the robustness, and in particular, a risk dominated equilibrium is shown to be robust in $\left(\mathcal{G}, \mathcal{T}^{c}\right)$.

\section{5 p-Dominance and robustness}

\section{1 p-Dominance}

Let us denote $\mathbf{p}=\left(p_{1}, p_{2}, \ldots, p_{I}\right) \in[0,1]^{I}$. We first introduce the standard $\mathbf{p}$ dominance in $\mathcal{G}$ (Morris et al., 1995; Kajii and Morris, 1997). Let $\phi_{i} \in \Delta\left(A_{-i}\right)$ for each $i \in \mathcal{I}$. An action profile $a^{*} \in A$ is $\mathbf{p}$-dominant in $\mathcal{G}$ if, for each $i \in$ $\mathcal{I}, a_{i} \in A_{i}$, and $\phi_{i} \in \Delta\left(A_{-i}\right)$ with $\phi_{i}\left(a_{-i}^{*}\right) \geq p_{i}$, we have $\sum_{a_{-i} \in A_{-i}}\left(u_{i}\left(a_{i}^{*}, a_{-i}\right)-\right.$ $\left.u_{i}\left(a_{i}, a_{-i}\right)\right) \phi_{i}\left(a_{-i}\right) \geq 0$. Thus, $a_{i}^{*}$ is a best response for player $i$ if he believes that the other players will play $a_{-i}^{*}$ with probability at least $p_{i}$.

We analogously define $\mathbf{p}$-dominance in $(\mathcal{G}, \mathcal{T})$. Let $\lambda_{i}\left(c_{i}\right) \in \Delta\left(C_{-i} \times A_{-i}\right)$ denote a belief of type $c_{i}$ about other players' types and actions, and write $\lambda_{i}\left(c_{i}\right)\left(c_{-i}, a_{-i}\right)$ for the probability assigned to $\left(c_{-i}, a_{-i}\right) \in C_{-i} \times A_{-i}$ under $\lambda_{i}\left(c_{i}\right)$. We say that $\lambda_{i}\left(c_{i}\right)$ is consistent if $\sum_{a_{-i} \in A_{-i}} \lambda_{i}\left(c_{i}\right)\left(\cdot, a_{-i}\right)=\mu_{i}\left(c_{i}\right)(\cdot)$.

Definition 5.1. A strategy profile $s^{*}$ is $\mathbf{p}$-dominant in $(\mathcal{G}, \mathcal{T})$ if, for each $i \in \mathcal{I}$, $c_{i} \in C_{i} \backslash\{0\}, a_{i} \in A_{i}$, and consistent $\lambda_{i}\left(c_{i}\right)$ with $\sum_{c_{-i} \in C_{-i}} \lambda_{i}\left(c_{i}\right)\left(c_{-i}, s_{-i}^{*}\left(c_{-i}\right)\right) \geq p_{i}$, we have

$$
\sum_{c_{-i} \in C_{-i}} \sum_{a_{-i} \in A_{-i}}\left(u_{i}\left(s_{i}^{*}\left(c_{i}\right), a_{-i}\right)-u_{i}\left(a_{i}, a_{-i}\right)\right) \lambda_{i}\left(c_{i}\right)\left(c_{-i}, a_{-i}\right) \geq 0 .
$$


This definition says that it is optimal for $c_{i}$ to play $s_{i}^{*}\left(c_{i}\right)$ if he believes consistently with $\mu_{i}\left(c_{i}\right)$ that the other players will play $s_{-i}^{*}$ with probability at least $p_{i}$. For instance, recall the coordinated attack model in Section 4. The model has a unique equilibrium in which all types choose $A$. Since $(A, A)$ is $2 / 3$-dominant, this equilibrium is $2 / 3$-dominant for any type space.

The following three properties of our p-dominance are all straightforward, and hence are stated without proofs. ${ }^{11}$

Proposition 5.1. In $(\mathcal{G}, \mathcal{T})$ :

(1) $s^{*}$ is an equilibrium if and only if it is 1-dominant;

(2) if $s^{*}$ is $\boldsymbol{p}$-dominant, it is $\boldsymbol{q}$-dominant for each $\boldsymbol{p} \leq \boldsymbol{q}{ }^{12}$

(3) $s^{*}$ is $\boldsymbol{p}$-dominant if all types of players follow a $\boldsymbol{p}$-dominant action profile $a^{*}$ in $\mathcal{G}$, that is, $s_{i}^{*}\left(c_{i}\right)=a_{i}^{*}$ for each $c_{i} \in C_{i}$ and $i \in \mathcal{I}$.

\subsection{Main result}

Here is our main result.

Theorem 5.1. A p-dominant equilibrium of $(\mathcal{G}, \mathcal{T})$ is robust if there exists a threshold level $\bar{c} \in \mathbb{N}$ such that, for each $i \in \mathcal{I}$,

$$
\sup _{c_{i}>\bar{c}} \sum_{\left\{c_{-i}: \bar{c} \leq c_{j}, \exists j \neq i\right\}} \mu_{i}\left(c_{i}\right)\left(c_{-i}\right)<\frac{I\left(1-p_{i}\right)}{I-1} .
$$

Proof. See the Appendix.

This condition is satisfied if there is a certain threshold level $\bar{c}$ such that all higher-level types than $\bar{c}$ put sufficiently small probability (decreasing in $\mathbf{p}$ ) on some other players having a level higher than or equal to $\bar{c}$. With the level-k type space, this condition reduces to " $p_{i}<1 / I$ for each $i \in \mathcal{I}$." In contrast, if there is a

\footnotetext{
${ }^{11}$ Unlike the standard $\mathbf{p}$-dominance, there does not necessarily exist some $\mathbf{p} \in[0,1)^{I}$ that makes a strict equilibrium p-dominant. To avoid this, we can generalize $\mathbf{p}$-dominance by allowing each probability $p_{i}$ to depend on each type $c_{i}$. With some notational complication, this generalization gives us a slightly stronger result.

${ }^{12} \mathbf{p} \leq \mathbf{q}$ means $p_{i} \leq q_{i}$ for each $i \in \mathcal{I}$.
} 
probability distribution over reasoning levels, the sum of conditional probabilities on slightly lower-level types vanishes as a player's reasoning level increases. Thus, the $\mathrm{CH}$ type space satisfies the inequality for each $p_{i} \in[0,1)$. Consequently, the two prominent type spaces exhibit a great difference in the existence of robust equilibria.

Corollary 5.1. A p-dominant equilibrium of $\left(\mathcal{G}, \mathcal{T}^{k}\right)$ is robust if $p_{i}<1 / I$ for each $i \in \mathcal{I}$.

Corollary 5.2. A $\boldsymbol{p}$-dominant equilibrium of $\left(\mathcal{G}, \mathcal{T}^{c}\right)$ is robust for each $\boldsymbol{p} \in[0,1)^{I}$.

As demonstrated in the existing literature, non-robustness is often caused by the contagion argument through higher-order beliefs. Intuitively, player's FDR restricts the dependence of his best response on higher-order beliefs. Hence, the behavior of low-level types continuously changes in any close model. However, the impact of higher-order beliefs can be significant if player's reasoning level is high because the behavior of Lk type depends on the behavior of Lk-1 type, which in turn depends on the behavior of Lk-2 type, and so on. Our main result shows that such higherorder dependence can be (uniformly) restricted by an upper-bound on the first-order beliefs of high-level types.

Theorem 5.1 is closely related to Theorem 4 of Strzalecki (2014), which gives an upper-bound on beliefs to make the risk dominated equilibrium continuously played in the email game. Our result further develops his insight that beliefs about the opponents' reasoning levels have a significant impact on players' strategic interaction. A main difference is that our bound is uniform to all the close models (including the email game elaboration), and thus it does not depend on the details of information structure. Instead, our bound is a function of the "strength" of equilibrium (p) and the number of players $(I)$. As a result, the bound also tells us the threshold value of $\mathbf{p}$ that makes an equilibrium robust for each type space. In addition, as the number of players increases, the bound becomes tighter so that we need a stronger restriction on beliefs to have a robust equilibrium.

Theorem 5.1 is also related to Proposition 5.3 of Kajii and Morris (1997), which shows that a $\mathbf{p}$-dominant action profile is $\mathrm{KM}$ robust if $\sum_{i \in \mathcal{I}} p_{i}<1$. In particular, when $\mathbf{p}=(p, \ldots, p)$, their condition is stronger than ours. On the other hand, 
the relation is reversed when we have the level-k type space. Hence, there is no clear logical relation between two results. However, we can directly show that a p-dominant equilibrium is robust under the Kajii and Morris' condition.

Proposition 5.2. A p-dominant equilibrium of $(\mathcal{G}, \mathcal{T})$ is robust if $\sum_{i \in \mathcal{I}} p_{i}<1$.

Proof. See the Appendix.

\section{Dominance solvability and robustness}

Our second sufficient condition relates dominance solvability to robustness. First, we define the dominance solvability of $(\mathcal{G}, \mathcal{T})$. For L0 types, set $S_{i}^{n}(0)=A_{i}$ for each $n \geq 0$. For other types, let $S_{i}^{0}(k)=A_{i}$, and define $S_{i}^{n}(k)$ recursively by $a_{i} \in S_{i}^{n}(k)$ if and only if

$$
a_{i} \in \arg \max _{a_{i}^{\prime}} \sum_{c_{-i} \in C_{-i}} \sum_{a_{-i} \in A_{-i}} u_{i}\left(a_{i}^{\prime}, a_{-i}\right) \lambda_{i}\left(c_{-i}, a_{-i}\right)
$$

for some $\lambda_{i} \in \Delta\left(C_{-i} \times A_{-i}\right)$ such that $\sum_{a_{-i} \in A_{-i}} \lambda_{i}\left(\cdot, a_{-i}\right)=\mu_{i}(k)(\cdot)$ and $\lambda_{i}\left(c_{-i}, a_{-i}\right)>$ 0 only if $a_{j} \in S_{j}^{n-1}\left(c_{j}\right)$ for each $j \neq i$. We write $S_{i}^{\infty}(k)=\bigcap_{n \geq 1} S_{i}^{n}(k)$.

Definition 6.1. $(\mathcal{G}, \mathcal{T})$ is dominance solvable if there exists $n \in \mathbb{N}$ such that $\left|S_{i}^{\infty}(k)\right|=1$ for each $k \geq n$ and $i \in \mathcal{I}$.

The set $S_{i}^{\infty}(k)$ corresponds to a set of (interim correlated) rationalizable actions for the Lk type of player $i$ (Bernheim 1984; Pearce 1984; Dekel et al. 2007). Hence, a model is dominance solvable if and only if sufficiently high-level types have a unique rationalizable action. Since the rationalizability is clearly not a valid solution concept for our model, it is important to note that we introduce dominance solvability to hypothetically consider the implication of having differently modeled anchor behaviors.

By definition, if $(\mathcal{G}, \mathcal{T})$ is dominance solvable, $\mathcal{G}$ is dominance solvable in the usual sense. While the converse is also true with the level-k type space, this does not hold in general as the following example shows.

Example 6.1. Consider the following game given by Table 6 with arbitrarily chosen anchor behavior. First, this game is dominance solvable with a unique rationalizable 


\begin{tabular}{ccc}
\hline \hline & $A_{2}$ & $B_{2}$ \\
\hline$A_{1}$ & 2,2 & 1,1 \\
\hline$B_{1}$ & 1,2 & 5,1 \\
\hline
\end{tabular}

Table 6: Payoff matrix.

action pair $\left(A_{1}, A_{2}\right)$. Let us introduce the $\mathrm{CH}$ type space with a Poisson distribution of parameter 1.5. ${ }^{13}$ Since each type of player 1 believes that player 2 is of L0 type with probability at least $1 / 5$, we have $S_{1}^{\infty}(k)=\left\{A_{1}, B_{1}\right\}$ and $S_{2}^{\infty}(k)=\left\{A_{2}\right\}$ for each $k \geq 1$. Thus, this model is not dominance solvable.

In a dominance solvable model, changes in anchor behavior have no effect on the equilibrium behavior of high-level types. That is, even if we replace the original anchor behavior with any other action, all the modified models yield the same equilibrium action for sufficiently high-level types. This straightforward observation leads to the following proposition.

Proposition 6.1. A strict equilibrium of a dominance solvable model is robust.

Proof. See the Appendix.

Remark 6.1. The strictness of an equilibrium cannot be dropped because low-level types may change their behavior dramatically otherwise. Consider the $3 \times 3$ game given by Table 7 with $f_{i}=C_{i}$ for each $i=1,2$, and consider the level-k type space. It

\begin{tabular}{cccc}
\hline \hline & $A_{2}$ & $B_{2}$ & $C_{2}$ \\
\hline$A_{1}$ & 2,2 & 1,1 & 1,0 \\
\hline$B_{1}$ & 1,2 & 1,1 & 1,0 \\
\hline$C_{1}$ & 0,1 & 0,1 & 1,0 \\
\hline
\end{tabular}

Table 7: Payoff matrix.

is easy to check that this model is dominance solvable. This model has an equilibrium $s^{*}$ such that $s_{1}^{*}=\left(C_{1}, B_{1}, B_{1}, A_{1}, A_{1}, \ldots\right)$, and $s_{2}^{*}=\left(C_{2}, B_{2}, A_{2}, A_{2}, \ldots\right)$. Let us introduce the following $\varepsilon$-elaboration: $\Theta=\left\{\theta_{1}, \theta_{2}, \theta_{3}, \theta_{4}\right\}, \Pi_{1}=\left\{\left\{\theta_{1}\right\},\left\{\theta_{2}, \theta_{3}, \theta_{4}\right\}\right\}$,

\footnotetext{
${ }^{13}$ Camerer et al. (2004) report that the median estimate of the Poisson parameter is 1.61 in 24 beauty contest games.
} 
$\Pi_{2}=\left\{\left\{\theta_{1}, \theta_{2}\right\},\left\{\theta_{3}, \theta_{4}\right\}\right\}, P\left(\theta_{1}\right)=P\left(\theta_{2}\right)=\varepsilon / 2$, and $P\left(\theta_{3}\right)=P\left(\theta_{4}\right)=(1-\varepsilon) / 2$. Suppose that player 1's anchor behavior is $A_{1}$ at $\theta_{1}$ instead of $C_{1}$. Then $A_{2}$ is a unique best response in $\left\{\theta_{1}, \theta_{2}\right\}$ for the L1 type of player 2 . Therefore, $A_{1}\left(\neq s_{1}^{*}(2)\right)$ is a unique best response at any state for the L2 type of player 1 (see Table 8). Hence, $s^{*}$ is not a robust equilibrium.

\begin{tabular}{|c|c|c|c|c|}
\hline$c_{1}=2$ & $A_{1}$ & \multicolumn{3}{|c|}{$A_{1}$} \\
\hline$c_{1}=1$ & & & & \\
\hline \multirow[t]{2}{*}{$c_{1}=0$} & $A_{1}$ & & $C_{1}$ & \\
\hline & $\theta_{1}$ & $\theta_{2}$ & $\theta_{3}$ & \\
\hline$c_{2}=0$ & & & & $C_{2}$ \\
\hline$c_{2}=1$ & & & & \\
\hline
\end{tabular}

Table 8: Equilibrium actions of low-level types.

In the experimental literature, dominance solvable games such as the $2 / 3$-guessing game are often used. As long as we stick to such a class of games and the level-k type space, our robustness has little bite as the following corollary shows.

Corollary 6.1. A strict equilibrium of $\left(\mathcal{G}, \mathcal{T}^{k}\right)$ is robust if $\mathcal{G}$ is dominance solvable.

\section{Discussion}

This paper considers the robustness of equilibria in FDR models. An equilibrium is robust if it can be played with high probability in any close model where the anchor behavior remains the same and players know that with high probability. We provide two different sufficient conditions for the robustness: any p-dominant equilibrium satisfying a certain condition on beliefs, and any strict equilibrium of dominance solvable models.

One may think that our robustness is less appealing because experimental evidence suggests that people have a very low depth of reasoning. However, notice the fact that lab experiments usually exclude experienced players from their subjects. In addition, learning from past experiences improves reasoning ability, and often 
leads to an equilibrium play. ${ }^{14}$ Hence, beyond the ideally controlled environment, we have no strong evidence against the existence of players who can reason almost indefinitely. Our result is especially meaningful when analysts cannot exclude the possibility of players being in such an equilibration process.

Finally, an implicit assumption of this paper is that each player has no difficulty in modeling a complicated anchor behavior. ${ }^{15}$ Some may argue that players, who are bounded rational as in the FDR model, may not describe anchor behavior to be as complex as those in the email game elaboration. However, we think that player's FDR and the limited ability of modeling anchor behavior are two different concepts. This paper focuses on the former to examine its implication as clearly as possible. Future work may investigate a unified framework that accommodates both concepts of bounded rationality.

\section{Appendix: Omitted proofs}

\section{Proof of Theorem 5.1}

Proof. Take any $\mathbf{p}$-dominant equilibrium $s^{*}$ of $(\mathcal{G}, \mathcal{T})$. If $p_{i}=1$ for some $i$, then the theorem vacuously holds. Suppose $\mathbf{p} \in[0,1)^{\mathcal{I}}$ in the sequel. Fix any $\varepsilon>0$ and $\varepsilon$-elaboration $\mathcal{U} \in E(\mathcal{G}, \varepsilon)$. The proof consists of four steps.

\section{Step 1. Construct events in which $s^{*}$ is played in equilibrium.}

Let $E_{(i, 0)} \equiv \Omega_{\mathcal{U}}$ for each $i \in \mathcal{I}$, and inductively define $E_{(i, k)}$ as follows:

$$
E_{(i, k)} \equiv\left\{\theta \in \Theta: \sum_{\left\{c_{-i}: c_{j}<k, \forall j \neq i\right\}} \mu_{i}(k)\left(c_{-i}\right) P\left(E_{c_{-i}} \mid \pi_{i}(\theta)\right) \geq p_{i}\right\} \cap E_{\mathbf{0}}
$$

for each $k>0$ and $i \in \mathcal{I}$, where $E_{c_{-i}}=\bigcap_{j \neq i} E_{\left(j, c_{j}\right)}$ and $E_{\mathbf{0}}=\Omega_{\mathcal{U}}$. The following lemma shows that this is the event we are looking for.

Lemma A.1. $(\mathcal{U}, \mathcal{T})$ has an equilibrium in which the Lk type of player $i$ plays $s_{i}^{*}(k)$ in $E_{(i, k)}$ for each $k \geq 0$ and $i \in \mathcal{I}$.

\footnotetext{
${ }^{14}$ In fact, Ho et al. (2013) and Ho and $\mathrm{Su}$ (2013) propose models that allow players in FDR models to update their reasoning levels and beliefs.

${ }^{15}$ I thank Michihiro Kandori for pointing out this issue.
} 
Proof. This proof proceeds similarly to Lemma 5.2 of Kajii and Morris (1997). Since $E_{(i, 0)}=\Omega_{\mathcal{U}}$ for each $i \in \mathcal{I}$, we have $\sigma_{i}(0, \theta)=s_{i}^{*}(0)$ for each $\theta \in E_{(i, 0)}$ and $i \in \mathcal{I}$. Let us construct the modified model of $(\mathcal{U}, \mathcal{T})$ where each player's strategy is restricted as follows: $\sigma_{i}(k, \theta)=s_{i}^{*}(k)$ for each $\theta \in E_{(i, k)}, k>0$, and $i \in \mathcal{I}$. That is, in the modified model, the Lk type of player $i$ can freely choose his action in $\Theta \backslash E_{(i, k)}$; however, he must choose $\sigma_{i}(k, \theta)=s_{i}^{*}(k)$ at any $\theta \in E_{(i, k)}$. Clearly, each restricted strategy of the modified game can be considered as a strategy of $(\mathcal{U}, \mathcal{T})$. We can find an "equilibrium" $\sigma^{*}$ of this modified model such that $\sigma_{i}^{*}(k, \theta)$ satisfies the equilibrium condition in $(\mathcal{U}, \mathcal{T})$ for each $\theta \in \Theta \backslash E_{(i, k)}, k>0$, and $i \in \mathcal{I}$. We show that $\sigma^{*}$ is also an equilibrium of $(\mathcal{U}, \mathcal{T})$. Let us consider the Lk type of player $i$. By definition, $\sigma_{i}^{*}(k, \theta)$ is a best response to $\sigma_{-i}^{*}$ at any $\theta \in \Theta \backslash E_{(i, k)}$. It remains to show the case of $\theta \in E_{(i, k)}$. Let us introduce $\lambda_{i}(k) \in \Delta\left(C_{-i} \times A_{-i}\right)$ such that for each $c_{-i} \in C_{-i}$ and $a_{-i} \in A_{-i}, \lambda_{i}(k)\left(c_{-i}, a_{-i}\right)=\mu_{i}(k)\left(c_{-i}\right) \cdot P\left(\left\{\theta^{\prime} \in \Theta: \sigma_{-i}^{*}\left(c_{-i}, \theta^{\prime}\right)=a_{-i}\right\} \mid \pi_{i}(\theta)\right)$. Then by our construction of $\sigma^{*}$, we have

$$
\begin{aligned}
& \sum_{c_{-i} \in C_{-i}} \lambda_{i}\left(c_{i}\right)\left(c_{-i}, s_{-i}^{*}\left(c_{-i}\right)\right) \\
& \quad=\sum_{c_{-i} \in C_{-i}} \mu_{i}(k)\left(c_{-i}\right) \cdot P\left(\left\{\theta^{\prime} \in \Theta: \sigma_{-i}^{*}\left(c_{-i}, \theta^{\prime}\right)=s_{-i}^{*}\left(c_{-i}\right)\right\} \mid \pi_{i}(\theta)\right) \\
& \quad \geq \sum_{c_{-i} \in C_{-i}} \mu_{i}(k)\left(c_{-i}\right) \cdot P\left(E_{c_{-i}} \mid \pi_{i}(\theta)\right) \geq p_{i} .
\end{aligned}
$$

Since $s^{*}$ is $\mathbf{p}$-dominant in $(\mathcal{G}, \mathcal{T})$, it follows that for each $a_{i} \in A_{i}$,

$$
\begin{aligned}
\sum_{c_{-i} \in C_{-i}} \sum_{\theta^{\prime} \in \Theta} u_{i}\left(s_{i}^{*}(k), \sigma_{-i}^{*}\left(c_{-i}, \theta^{\prime}\right)\right) \mu_{i}(k)\left(c_{-i}\right) P\left(\theta^{\prime} \mid \pi_{i}(\theta)\right) & \\
& =\sum_{c_{-i} \in C_{-i}} \sum_{a_{-i} \in A_{-i}} u_{i}\left(s_{i}^{*}(k), a_{-i}\right) \lambda_{i}(k)\left(c_{-i}, a_{-i}\right) \\
& \geq \sum_{c_{-i} \in C_{-i}} \sum_{a_{-i} \in A_{-i}} u_{i}\left(a_{i}, a_{-i}\right) \lambda_{i}(k)\left(c_{-i}, a_{-i}\right) \\
& =\sum_{c_{-i} \in C_{-i}} \sum_{\theta^{\prime} \in \Theta} u_{i}\left(a_{i}, \sigma_{-i}^{*}\left(c_{-i}, \theta^{\prime}\right)\right) \mu_{i}(k)\left(c_{-i}\right) P\left(\theta^{\prime} \mid \pi_{i}(\theta)\right) .
\end{aligned}
$$

Thus, $s_{i}^{*}(k, \theta)$ is a best response to $\sigma_{-i}^{*}$ in $E_{(i, k)}$. Therefore, $\sigma^{*}$ is an equilibrium of $(\mathcal{U}, \mathcal{T})$ that satisfies the desired property.

Step 2. Find a lower-bound for each $P\left(E_{(i, k)}\right)$.

First, we introduce the notion of common p-belief following Monderer and Samet 
(1989). Let $\mathcal{F}_{i}$ denote a $\sigma$-algebra generated by $\Pi_{i}$ for each $i \in \mathcal{I}$. The $p$-belief operator is defined by: $B_{i}^{p}(E) \equiv\left\{\theta \in \Theta: P\left(E \mid \pi_{i}(\theta)\right) \geq p\right\}$ for each (measurable) event $E$. Thus, $B_{i}^{p}(E)$ is a set of states in which player $i$ believes with probability at least $p$ that the event $E$ occurs. For each $\mathbf{p} \in[0,1]^{I}$, let us write $B_{*}^{\mathbf{p}}(E)=$ $\bigcap_{i \in \mathcal{I}} B_{i}^{p_{i}}(E)$, and $\left[B_{*}^{\mathbf{p}}\right]^{n}(E)=B_{*}^{\mathbf{p}}\left(\left[B_{*}^{\mathbf{p}}\right]^{n-1}(E)\right)$ for each $n \in \mathbb{N}$, where $\left[B_{*}^{\mathbf{p}}\right]^{0}(E)=E$. We say that an event $E$ is common p-belief at $\theta$ if, at $\theta, E$ is believed by each player with probability at least $p_{i}$, it is believed by each player with probability at least $p_{i}$ that $E$ is believed by each player with probability at least $p_{i}$, and so on, ad infinitum.

Definition A.1. An event $E$ is common $\boldsymbol{p}$-belief at $\theta$ if $\theta \in C^{\mathbf{p}}(E) \equiv \bigcap_{n \geq 1}\left[B_{*}^{\mathbf{p}}\right]^{n}(E)$.

An event $E$ is said to be simple if $E=\bigcap_{i \in \mathcal{I}} E_{i}$ with $E_{i} \in \mathcal{F}_{i}$ for each $i \in \mathcal{I}$. For instance, the event $\Omega_{\mathcal{U}}$ is simple. Kajii and Morris (1997) uncover a surprising relationship between the probability of a simple event and the probability of an event in which that simple event is common $\mathbf{p}$-belief:

Lemma A.2. (Proposition 4.2 of Kajii and Morris 1997) If $\sum_{i \in \mathcal{I}} p_{i}<1$, any simple event $E$ satisfies

$$
P\left(C^{\mathbf{p}}(E)\right) \geq 1-(1-P(E))\left(\frac{1-\min _{i \in \mathcal{I}} p_{i}}{1-\sum_{i \in \mathcal{I}} p_{i}}\right) .
$$

The following lemma gives a loose lower-bound for the probability of an event $\left[B_{*}^{\mathbf{p}}\right]^{n}(E) .{ }^{16}$

Lemma A.3. (Lemma B of Kajii and Morris 1997)

For each $\boldsymbol{p} \in[0,1)^{I}$ and event $E$, we have

$$
P\left(\left[B_{*}^{\mathbf{p}}\right]^{n}(E)\right) \geq 1-\left(1+\sum_{i \in \mathcal{I}} \frac{p_{i}}{1-p_{i}}\right)^{n}(1-P(E)) .
$$

When we have the level-k type space, $E_{(i, k)}$ can be written as $E_{(i, k)}=\{\theta \in \Theta$ : $\left.P\left(E_{c_{-i}} \mid \pi_{i}(\theta)\right) \geq p_{i}\right\} \cap E_{\mathbf{0}}=B_{i}^{p_{i}}\left(E_{c_{-i}}\right) \cap E_{\mathbf{0}}$, where $c_{j}=k-1$ for each $j \neq i$. Let us denote this $E_{(i, k)}$ by $\widehat{E}_{(i, k)}$. The following lemma provides three properties of $\widehat{E}_{(i, k)}$.

Lemma A.4. The following three properties hold.

\footnotetext{
${ }^{16}$ This bound is loose since it goes $-\infty$ as $n \rightarrow \infty$ whenever $P(E)<1$.
} 
(1) $\widehat{E}_{(i, k)}$ is decreasing in $k$;

(2) $\widehat{E}_{(i, k)} \subseteq E_{(i, k)}$;

(3) $\left[B_{*}^{p}\right]^{k}\left(E_{\boldsymbol{o}}\right) \subseteq \bigcap_{i \in \mathcal{I}} \widehat{E}_{(i, k)}$

Proof. All of the three properties are shown by mathematical induction.

(1) If $k=0, \widehat{E}_{(i, 1)}=B_{i}^{p_{i}}\left(E_{\mathbf{0}}\right) \cap E_{\mathbf{0}} \subseteq E_{\mathbf{0}}=\widehat{E}_{(i, 0)}$. Suppose $\widehat{E}_{(i, k+1)} \subseteq \widehat{E}_{(i, k)}$ for each $i \in \mathcal{I}$. Then $\widehat{E}_{(i, k+2)}=B_{i}^{p_{i}}\left(\bigcap_{j \neq i} \widehat{E}_{(j, k+1)}\right) \cap E_{\mathbf{0}} \subseteq B_{i}^{p_{i}}\left(\bigcap_{j \neq i} \widehat{E}_{(j, k)}\right) \cap E_{\mathbf{0}}=\widehat{E}_{(i, k+1)} . \|$

(2) By definition, $E_{(i, 0)}=\widehat{E}_{(i, 0)}=E_{\mathbf{0}}$. Suppose that the claim holds up to $\mathrm{k}$ for each $i \in \mathcal{I}$. Then since $\widehat{E}_{\left(j, c_{j}\right)} \subseteq E_{\left(j, c_{j}\right)}$ for each $0 \leq c_{j} \leq k$ and $j \neq i$, we have $E_{(i, k+1)} \supseteq\left\{\theta \in \Theta: \sum_{c_{-i} \in C_{-i}} \mu_{i}(k+1)\left(c_{-i}\right) P\left(\widehat{E}_{c_{-i}} \mid \pi_{i}(\theta)\right) \geq p_{i}\right\} \cap E_{\mathbf{0}} \supseteq\{\theta \in \Theta:$ $\left.P\left(\bigcap_{j \neq i} \widehat{E}_{(j, k)} \mid \pi_{i}(\theta)\right) \geq p_{i}\right\} \cap E_{\mathbf{0}}=\widehat{E}_{(i, k+1)}$. The second inclusion follows by (1). \|

(3) This holds if $k=0$. Suppose $\left[B_{*}^{\mathbf{p}}\right]^{k}\left(E_{\mathbf{0}}\right) \subseteq \bigcap_{i \in \mathcal{I}} \widehat{E}_{(i, k)}$. Then we have $\widehat{E}_{(i, k+1)}=$ $B_{i}^{p_{i}}\left(\bigcap_{j \neq i} \widehat{E}_{(j, k)}\right) \cap E_{\mathbf{0}} \supseteq B_{i}^{p_{i}}\left(\left[B_{*}^{\mathbf{p}}\right]^{k}\left(E_{\mathbf{0}}\right)\right) \cap E_{\mathbf{0}}$. Since $E_{\mathbf{0}}$ is simple, we have $\bigcap_{i \in \mathcal{I}} \widehat{E}_{(i, k+1)} \supseteq$ $\bigcap_{i \in \mathcal{I}} B_{i}^{p_{i}}\left(\left[B_{*}^{\mathbf{p}}\right]^{k}\left(E_{\mathbf{0}}\right)\right) \cap E_{\mathbf{0}}=\left[B_{*}^{\mathbf{p}}\right]^{k+1}\left(E_{\mathbf{0}}\right) . \|$

Combining above results gives a lower-bound for $P\left(E_{(i, k)}\right)$.

Step 3. Find a tighter lower-bound for $P\left(E_{(i, k)}\right)$ with $k \geq \bar{c}$.

By our assumption, there exists $\bar{c} \in \mathbb{N}$ such that $\sup _{k>\bar{c}} \sum_{\left\{c_{-i}: \bar{c} \leq c_{j}, \exists j \neq i\right\}} \mu_{i}(k)\left(c_{-i}\right)<$ $I\left(1-p_{i}\right) /(I-1)$ for each $i \in \mathcal{I}$. By definition of the supremum, we can take $\omega>0$ such that $\sum_{\left\{c_{-i}: \bar{c} \leq c_{j}, \exists j \neq i\right\}} \mu_{i}(k)\left(c_{-i}\right)<\left(I-(I+\omega) p_{i}\right) /(I-1)$ for each $k>\bar{c}$ and $i \in \mathcal{I}$. Define $\varphi \equiv I /(I+\omega)$, and $\tau \equiv \varphi / I=1 /(I+\omega)$. Then we have $0<\varphi<1$, and $0<\tau<1 / I$. By using $\varphi$ and $\tau$, let us inductively construct an event $F_{(i, n)}$ for each $n=0,1, \ldots$ as follows:

$$
F_{(i, 0)}=F_{\mathbf{0}} \equiv B_{*}^{\varphi}\left(\bigcap_{i \in \mathcal{I}} \widehat{E}_{(i, \bar{c})}\right) \text {, and } F_{(i, n+1)} \equiv B_{i}^{\tau}\left(F_{n}\right) \cap F_{\mathbf{0}} \text { where } F_{n} \equiv \bigcap_{j \neq i} F_{(j, n)} \text {. }
$$

We show that $F_{(i, n)}$ is a subset of $E_{(i, \bar{c}+n)}$ under our hypothesis.

Lemma A.5. $F_{(i, n)} \subseteq E_{(i, \bar{c}+n)}$ for each $n \geq 0$ and $i \in \mathcal{I}$.

Proof. We show this lemma by induction. By the definition of $F_{(i, 0)}$, we have $F_{(i, 0)} \subseteq$ $E_{(i, \bar{c})}$ for each $i \in \mathcal{I}$. Suppose that the lemma holds up to $n$. Take any $\theta \in F_{(i, n+1)}$. 
Let $t=\bar{c}+n+1$. Then we have

$$
\begin{aligned}
& \sum_{c_{-i} \in C_{-i}} \mu_{i}(t)\left(c_{-i}\right) P\left(E_{c_{-i}} \mid \pi_{i}(\theta)\right) \\
& \quad=\sum_{\left\{c_{-i}: 0 \leq c_{j}<\bar{c}, \forall j \neq i\right\}} \mu_{i}(t)\left(c_{-i}\right) P\left(E_{c_{-i}} \mid \pi_{i}(\theta)\right)+\sum_{\left\{c_{-i}: \bar{c} \leq c_{j}, \exists j \neq i\right\}} \mu_{i}(t)\left(c_{-i}\right) P\left(E_{c_{-i}} \mid \pi_{i}(\theta)\right) \\
& \quad \geq \sum_{\left\{c_{-i}: 0 \leq c_{j}<\bar{c}, \forall j \neq i\right\}} \mu_{i}(t)\left(c_{-i}\right) P\left(\bigcap_{i \in \mathcal{I}} \widehat{E}_{(i, \bar{c})} \mid \pi_{i}(\theta)\right)+\sum_{\left\{c_{-i}: \bar{c} \leq c_{j}, \exists j \neq i\right\}} \mu_{i}(t)\left(c_{-i}\right) P\left(F_{n} \mid \pi_{i}(\theta)\right) \\
& \quad>\left(\left((I+\omega) p_{i}-1\right) /(I-1)\right) \cdot \varphi+\left(\left(I-(I+\omega) p_{i}\right) /(I-1)\right) \cdot \tau=p_{i} .
\end{aligned}
$$

The weak inequality follows since $F_{(i, n)}$ is decreasing in $n$, and hence $F_{(i, n)} \subseteq E_{(i, k)}$ for each $0 \leq k \leq \bar{c}+n$ by our induction hypothesis. The strict inequality follows since $\theta \in F_{\mathbf{0}} \subseteq B_{*}^{\varphi}\left(\bigcap_{i \in \mathcal{I}} \widehat{E}_{(i, \bar{c})}\right)$ and $\theta \in F_{(i, n+1)} \subseteq B_{i}^{\tau}\left(F_{n}\right)$. Thus, we have $\theta \in$ $E_{(i, \bar{c}+n+1)}$.

$P\left(F_{(i, n)}\right)$ gives a new lower-bound for $P\left(E_{(i, \bar{c}+n)}\right)$.

\section{Step 4. Combine the obtained results.}

Take any $\delta>0$, and let

$$
\varepsilon \leq \frac{\delta}{\left(1+\frac{I \varphi}{1-\varphi}\right)\left(1+\sum_{i \in \mathcal{I}} \frac{p_{i}}{1-p_{i}}\right)^{\bar{c}}\left(\frac{1-\tau}{1-I \tau}\right)} .
$$

Observe that by Lemmas A.3 and A.4, we have

$$
P\left(F_{\mathbf{0}}\right) \geq 1-\left(1+\frac{I \varphi}{1-\varphi}\right)\left(1-P\left(\bigcap_{i \in \mathcal{I}} \widehat{E}_{(i, \bar{c})}\right)\right) \geq 1-\varepsilon\left(1+\frac{I \varphi}{1-\varphi}\right)\left(1+\sum_{i \in \mathcal{I}} \frac{p_{i}}{1-p_{i}}\right)^{\bar{c}} .
$$

Then since $F_{n}$ is decreasing in $n, F_{(i, 0)} \subseteq E_{(i, k)}$ if $k \leq \bar{c}$, and $\sum_{i \in \mathcal{I}} \tau<1$, it follows from Lemmas A.2 and A.5 that for each type profile $c$,

$$
P\left(\bigcap_{i \in \mathcal{I}} E_{\left(i, c_{i}\right)}\right) \geq P\left(C^{\tau}\left(F_{\mathbf{0}}\right)\right) \geq 1-\left(1-P\left(F_{\mathbf{0}}\right)\right)\left(\frac{1-\tau}{1-I \tau}\right) \geq 1-\delta
$$

By Lemma A.1, there exists an equilibrium $\sigma^{*}$ of $(\mathcal{U}, \mathcal{T})$ with $P_{\sigma^{*}}\left(s^{*}(c) \mid c\right) \geq 1-\delta$ for each type profile $c$. Since $\mathcal{U}$ is arbitrary chosen from $E(\mathcal{G}, \varepsilon)$, we are done.

\section{Proof of Proposition 5.2}

Proof. Let $s^{*}$ denote a $\mathbf{p}$-dominant equilibrium of $(\mathcal{G}, \mathcal{T})$ with $\sum_{i \in \mathcal{I}} p_{i}<1$. Fix any $\delta>0$, and let $\varepsilon \leq \delta\left(1-\sum_{i \in \mathcal{I}} p_{i}\right) /\left(1-\min _{i \in \mathcal{I}} p_{i}\right)$. Take any $\mathcal{U} \in E(\mathcal{G}, \varepsilon)$. Fix any 
type profile $c$, and let $\hat{c}=\max _{i \in \mathcal{I}} c_{i}$. Since $\sum_{i \in \mathcal{I}} p_{i}<1$, it follows from Lemmas A.2 and A.4 that $P\left(\bigcap_{i \in \mathcal{I}} E_{\left(i, c_{i}\right)}\right) \geq P\left(\bigcap_{i \in \mathcal{I}} \widehat{E}_{(i, \hat{c})}\right) \geq P\left(C^{\mathbf{p}}\left(E_{\mathbf{0}}\right)\right) \geq 1-\delta$. Then by Lemma A.1, there exists an equilibrium $\sigma^{*}$ of $\mathcal{U}$ such that $P_{\sigma^{*}}\left(s^{*}(c) \mid c\right) \geq 1-\delta$, as desired.

\section{Proof of Proposition 6.1}

Proof. Let $s^{*}$ denote a strict equilibrium of $(\mathcal{G}, \mathcal{T})$. By our assumption, there exists $n \in \mathbb{N}$ such that $S_{i}^{\infty}(k)$ is a singleton for each $k \geq n$ and $i \in \mathcal{I}$. Since $s^{*}$ is a strict equilibrium, for each $i \in \mathcal{I}$ and $1 \leq k<n$, we can take $p_{i}^{k} \in[0,1)$ such that for each $a_{i} \in A_{i}$ and $\lambda_{i}(k) \in \Delta\left(C_{-i} \times A_{-i}\right)$ with $\sum_{a_{-i} \in A_{-i}} \lambda_{i}(k)\left(\cdot, a_{-i}\right)=\mu_{i}(k)(\cdot)$ and $\sum_{c_{-i} \in C_{-i}} \lambda_{i}(k)\left(c_{-i}, s_{-i}^{*}\left(c_{-i}\right)\right) \geq p_{i}^{k}$,

$$
\sum_{c_{-i} \in C_{-i}} \sum_{a_{-i} \in A_{-i}}\left(u_{i}\left(s_{i}^{*}(k), a_{-i}\right)-u_{i}\left(a_{i}, a_{-i}\right)\right) \lambda_{i}(k)\left(c_{-i}, a_{-i}\right) \geq 0 .
$$

Let $p_{i}=\max _{1 \leq k<n} p_{i}^{k}$ for each $i \in \mathcal{I}$. Fix any $\delta>0$, and let $\varepsilon \leq \delta /\left(1+\sum_{i \in \mathcal{I}} p_{i} /(1-\right.$ $\left.\left.p_{i}\right)\right)^{n-1}$. Consider any $\varepsilon$-elaboration $\mathcal{U} \in E(\mathcal{G}, \varepsilon)$. By using this $p_{i}$, construct the event $\widehat{E}_{(i, k)}$ for each $k>0$ as in the proof of Theorem 5.1. Then, by Lemmas A.1 and A.4, there exists an equilibrium $\sigma^{*}$ of $(\mathcal{U}, \mathcal{T})$ in which $s_{i}^{*}(k)$ is played in $\bigcap_{i \in \mathcal{I}} \widehat{E}_{(i, n-1)}$ by the Lk type of player $i$ for each $1 \leq k<n$ and $i \in \mathcal{I}$. By Lemmas A.3 and A.4, we have $P\left(\bigcap_{i \in \mathcal{I}} \widehat{E}_{(i, n-1)}\right) \geq P\left(\left[B_{*}^{\mathbf{p}}\right]^{n-1}\left(E_{\mathbf{0}}\right)\right) \geq 1-\delta$. For the behavior of higher-level types than $n-1$, we use the following claim.

Claim. $\sigma_{i}^{*}(k, \theta) \in S_{i}^{\infty}(k)$ for each $\theta \in \Theta, k>0$, and $i \in \mathcal{I}$.

Proof. This follows by translating a distribution over states into a distribution over other players' action profiles. Consider the Lk type of player $i$ and take any $\theta \in \Theta$. Since $\sigma^{*}$ is an equilibrium of $(\mathcal{U}, \mathcal{T})$, for each $a_{i} \in A_{i}$,

$$
\sum_{c_{-i} \in C_{-i}} \sum_{\theta^{\prime} \in \Theta}\left(u_{i}\left(\sigma_{i}^{*}(k, \theta), \sigma_{-i}^{*}\left(c_{-i}, \theta^{\prime}\right)\right)-u_{i}\left(a_{i}, \sigma_{-i}^{*}\left(c_{-i}, \theta^{\prime}\right)\right)\right) \mu_{i}(k)\left(c_{-i}\right) P\left(\theta^{\prime} \mid \pi_{i}(\theta)\right) \geq 0 .
$$

Let us define $\lambda_{i}^{\prime} \in \Delta\left(C_{-i} \times A_{-i}\right)$ such that for each $c_{-i} \in C_{-i}$ and $a_{-i} \in A_{-i}$, $\lambda_{i}^{\prime}\left(c_{-i}, a_{-i}\right)=\mu_{i}(k)\left(c_{-i}\right) \cdot P\left(\left\{\theta^{\prime} \in \Theta: \sigma_{-i}^{*}\left(c_{-i}, \theta^{\prime}\right)=a_{-i}\right\} \mid \pi_{i}(\theta)\right)$. Then the above inequality can be written as

$$
\sum_{c_{-i} \in C_{-i}} \sum_{a_{-i} \in A_{-i}}\left(u_{i}\left(\sigma_{i}^{*}(k, \theta), a_{-i}\right)-u_{i}\left(a_{i}, a_{-i}\right)\right) \lambda_{i}^{\prime}\left(c_{-i}, a_{-i}\right) \geq 0 \text { for each } a_{i} \in A_{i} .
$$


Hence, the claim is true for L1 types since $S_{j}^{\infty}(0)=A_{j}$ for each $j \neq i$. Suppose now that the claim holds up to $k-1$ for each $i \in \mathcal{I}$. Then we have $\lambda_{i}^{\prime}\left(c_{-i}, a_{-i}\right)>0$ only if $a_{j} \in S_{j}^{\infty}\left(c_{j}\right)$ for each $j \neq i$, that is, $\sigma_{i}^{*}(k, \theta) \in S_{i}^{\infty}(k)$. By our induction hypothesis, the claim follows. $\|$

Since $s_{i}^{*}(k) \in S_{i}^{\infty}(k)$ for each $k \geq 0$ and $i \in \mathcal{I}$, the above claim and our assumption imply that higher-level types than $n-1$ must follow $s^{*}$ in $\bigcap_{i \in \mathcal{I}} \widehat{E}_{(i, n-1)}$. Consequently, we have $P_{\sigma^{*}}\left(s^{*}(c) \mid c\right) \geq 1-\delta$ for each type profile $c$.

\section{References}

[1] Agranov, M., Caplin, A., and Tergiman, C. (2015). "Naive play and the process of choice in guessing game." Journal of Economic Science Association, forthcoming.

[2] Arad, A., and Rubinstein, A. (2012). "The 11-20 money request game: A level-k reasoning study." American Economic Review, 102, 3561-3573.

[3] Bernheim, B. D. (1984). "Rationalizable strategic behavior." Econometrica, 52, 1007-1028.

[4] Brandenburger, A., and Dekel, E. (1993). "Hierarchies of beliefs and common knowledge." Journal of Economic Theory, 59, 189-198.

[5] Burchardi, K., and Penczynski, S. (2014). "Out of your mind: Eliciting individual reasoning in one shot games." Games and Economic Behavior, 84, 39-57.

[6] Camerer, C. F., and Ho, T. H. (2014) "Behavioral game theory experiments and modeling." In H. P. Young and S Zamir (Ed.), Handbook of Game Theory Volume 4, 517- 574, North-Holland: Elsevier.

[7] Camerer, C. F., Ho, T. H., and Chong, J. K. (2004). "A cognitive hierarchy model of games." The Quarterly Journal of Economics, 119, 861-898.

[8] Carlsson, H., and van Damme, E. (1993). "Global games and equilibrium selection." Econometrica, 61, 989-1018. 
[9] Crawford, V. P. (2014). "A comment on "How portable is level-0 behavior? A test of level-k theory in games with non-neutral frames" by Heap, Rojo-Arjona, and Sugden." Mimeo, University of Oxford and University of California, San Diego.

[10] Crawford, V. P. (2015). "Efficient mechanisms for level-k bilateral trading" Mimeo, University of Oxford and University of California, San Diego.

[11] Crawford, V. P., Costa-Gomes, M. A., and Iriberri, N. (2013). "Structural models of nonequilibrium strategic thinking: Theory, evidence, and applications." Journal of Economic Literature, 51, 5-62.

[12] Crawford, V. P., Gneezy, U., and Rottenstreich, Y. (2008). "The power of focal points is limited: Even minute payoff asymmetry may yield large coordination failures." American Economic Review, 98, 1443-1458.

[13] Crawford, V. P., and Iriberri, N. (2007). "Level-k Auctions: Can a nonequilibrium model of strategic thinking explain the winner's curse and overbidding in private value auctions?" Econometrica, 75, 1721-1770.

[14] Crawford, V. P., Kugler, T., Neeman, Z., and Pauzner, A. (2009). "Behaviorally optimal auction design: Examples and observations." Journal of the European Economic Association, 7, 377-387.

[15] de Clippel, G., Saran, R., and Serrano, R. (2015). "Mechanism design with bounded depth of reasoning and small modeling mistakes." Mimeo, Brown University and Yale-NUS College.

[16] Dekel, E., Fudenberg, D., and Morris, S. (2007). "Interim correlated rationalizability." Theoretical Economics, 2, 15-40.

[17] Heap, S. H., Arjona, D. R., and Sugden, R. (2014). "How portable is level-0 behavior? A test of Level-k theory in games with non-neutral frames." Econometrica, 82, 1133-1151.

[18] Heifetz, A. and Kets, W. (2013). "Robust multiplicity with a grain of naiveté." CMS-EMS Discussion Paper \#1573, Northwestern University. 
[19] Ho, T. H., Park, S. E., and Su, X. (2013). "Level-r model with adaptive and sophisticated learning." Working paper, University of California, Berkeley.

[20] Ho, T. H., and Su, X. (2013). " Dynamic level-k model in sequential games." Management Science, 59, 452-469.

[21] Kajii, A., and Morris, S. (1997). "The robustness of equilibria to incomplete information." Econometrica, 65, 1283-1309.

[22] Kneeland, T. (2014). "Coordination under limited depth of reasoning." Mimeo, University College London.

[23] Kneeland, T. (2015). "Identifying higher-order rationality." Econometrica, forthcoming.

[24] Mertens, J. F., and Zamir, S. (1985). "Formulation of Bayesian analysis for games with incomplete information." International Journal of Game Theory, $14,1-29$.

[25] Monderer, D., and Samet, D. (1989). "Approximating common knowledge with common beliefs." Games and Economic Behavior, 1, 170-190.

[26] Morris, S., Rob, R., and Shin, H. S. (1995). "p-Dominance and belief potential." Econometrica, 63, 145-157.

[27] Morris, S., and Ui, T. (2005). "Generalized potentials and robust sets of equilibria." Journal of Economic Theory, 124, 24-78.

[28] Nagel, R. (1995). "Unraveling in guessing games: An experimental study." American Economic Review, 85, 1313-1326.

[29] Nora, V., and Uno, H. (2014). "Saddle functions and robust sets of equilibria." Journal of Economic Theory, 150, 866-877.

[30] Oyama, D., and Tercieux, O. (2009). "Iterated potential and robustness of equilibria." Journal of Economic Theory, 144, 1726-1769.

[31] Pearce, D. G. (1984), "Rationalizable strategic behavior and the problem of perfection." Econometrica, 52, 1029-1050. 
[32] Rubinstein, A. (1989). "The electronic mail game: Strategic behavior under "almost common knowledge"." American Economic Review, 79, 385-391.

[33] Schelling, T. C. (1960). The strategy of conflict. Cambridge: Harvard University Press.

[34] Stahl, D. O., and Wilson, P. W. (1994). "Experimental evidence on players' models of other players." Journal of Economic Behavior and Organization, 25, 309-327.

[35] Strzalecki, T. (2014). "Depth of reasoning and higher order beliefs." Journal of Economic Behavior and Organization, 108, 108-122.

[36] Tercieux, O. (2006). "p-Best response set and the robustness of equilibria to incomplete information." Games and Economic Behavior, 56, 371-384.

[37] Ui, T. (2001). "Robust equilibria of potential games." Econometrica, 69, 13731380.

[38] Weinstein, J., and Yildiz, M. (2007) "A structure theorem for rationalizability with application to robust predictions of refinement." Econometrica, 75, 365400. 